\title{
What is the Best Beam Energy for X-Ray Microanalysis of Nanomaterials in Electron Microscopy?
}

Raynald Gauvin ${ }^{1}$, Pierre Michaud ${ }^{1}$ and Michel L. Trudeau ${ }^{2}$

1. Department of Materials Engineering, McGill University, M. H. Wong Bldg, 3610 University Street, Montreal, Québec, Canada, H3A 2B2.

2. Chimie et Matériaux, Centre de Recherche d'Hydro-Québec, 1800 Boul. LionelBoulet, Varennes, Québec, Canada, J3X 1S1.

Electron microscopy has two main areas of applications, low voltage field emission scanning electron microscopy and high voltage field emission transmission electron microscopy with $C_{S}$ correctors. In the first case, the reduction of the electron beam energy leads to improved spatial resolution for $\mathrm{x}$-ray microanalysis but when the beam energy is too low, the yield of generated $x$-rays becomes too small. In the second case, a probe diameter of the order of $1 \AA$ with high current can be obtained with a corrector. Coupled with the high energy of the electron beam that penetrates almost in a straight line through the transparent material, high spatial resolution can be achieved. However, beam damage and specimen preparation issues are still concerns. However, the FIB has helped in fixing problems related to specimen preparation for FE-TEM. But, since the FIB allows making thin films of different materials, STEM imaging in dual beam FIB of in FESEM are now becoming a reality, as well as $x$-ray microanalysis in these conditions. Therefore, we now have to see $x$-ray microanalysis as a characterization technique that can be used between 3 to $300 \mathrm{keV}$ of electron beam energy. This raise the question of the best energy for $x$-ray microanalysis for a given material of fixed geometry and composition in terms of spatial resolution, $x$-ray count rate and peak to background ratio. This paper will discuss these issues as well as the problems that will have to be solved for performing quantitative $\mathrm{x}$-ray microanalysis of transparent materials with significant energy loss and beam spreading. It is obvious that quantitative $\mathrm{x}$-ray microanalysis in these conditions will be based on Monte Carlo simulations of electron trajectories. In the following figures, $x$-ray maps and line scans of a $10 \mathrm{~nm}$ cube of $\mathrm{Al}$ embedded in a cubic shell of Fe with $5 \mathrm{~nm}$ thickness are shown. These $256 \times 256$ maps were simulated at 5 and $200 \mathrm{keV}$ with 33 electron per pixel and $1 \mathrm{~nm}$ probe diameter using the new Monte Carlo program MC X-Ray that is described in another paper of this conference. The improvement of the spatial resolution at $200 \mathrm{keV}$ is obvious but performing $\mathrm{x}$-ray microanalysis at $5 \mathrm{keV}$ seems to be a viable option too. 


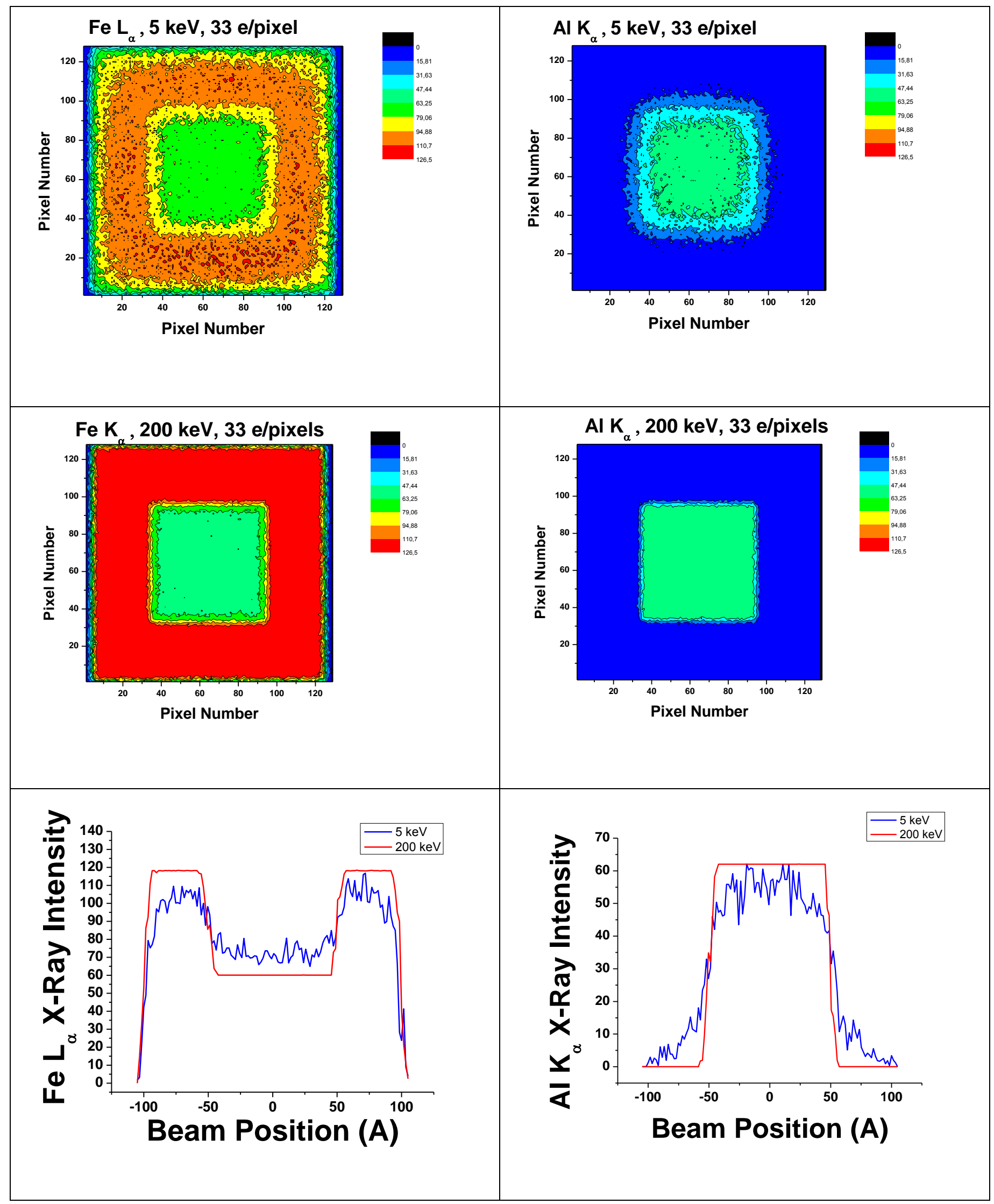

